\title{
Paleodemografía prehistórica: reflexiones sobre la metodología aplicada al estudio de algunos sepulcros colectivos de la Península Ibérica
}

\section{Prehistoric paleodemography: A methodological review based on the study of some collective tombs in the Iberian Peninsula}

\author{
ANGÉLICA SANTA CRUZ DEL BARRIO \\ Departamento de Prehistoria, Arqueología, Antropología Social y Ciencias y Técnicas \\ Historiográficas, Facultad de Filosofía y Letras, Universidad de Valladolid, Plaza del \\ Campus s/n, 47011 Valladolid \\ Email: angelica.santa-cruz@uva.es \\ ORCID: https://orcid.org/0000-0001-6186-0328
}

\section{PAZ CALDUCH BARDOLL}

Departamento de Medicina Legal, Toxicología y Antropología Física. Facultad de Medicina, Laboratorio de Antropología. Avenida de la Investigación, 11, Torre A, $4^{\mathrm{a}}$ planta, 18016 Granada

Email: paz.calduch@gmail.com

ORCID: https://orcid.org/0000-0001-6472-4313

Recibido: 06/06/2019. Aceptado: 01/07/2019.

Cómo citar: Santa Cruz, Angélica y Calduch, Paz (2018): "Paleodemografía prehistórica: reflexiones sobre la metodología aplicada al estudio de algunos sepulcros colectivos de la Península Ibérica”. BSAA arqueología, LXXXIV, pp. 71-93.

DOI: https://doi.org/10.24197/ba.LXXXIV.2018.71-93

Resumen: El análisis antropológico de los restos humanos es indisociable del estudio de cualquier estructura funeraria, pues los estudios bioarqueológicos ofrecen enormes posibilidades explicativas sobre las poblaciones del pasado $y$, en particular, de su régimen demográfico. Tratando de ahondar en esta cuestión, en este trabajo se analizan distintas propuestas paleodemográficas de sepulturas colectivas o conjuntos funerarios de la Prehistoria Reciente, quizás los contextos donde mayores dificultades encuentran este tipo de estudios. Se persigue con ello poner de relieve y comparar las soluciones metodológicas e interpretativas que se han propuesto para los estudios seleccionados. En cualquier caso, en todos ellos se evidencia que el análisis demográfico aplicado a los sepulcros colectivos en la Prehistoria Reciente ofrece una lectura más completa de estos espacios mortuorios a la vez que brinda la posibilidad de afrontar comparaciones basadas en la unidad de método.

Palabras clave: paleodemografía; Prehistoria Reciente; sepulcros colectivos; Península Ibérica 


\begin{abstract}
The anthropological analysis of human remains is indissociable from the study of any funerary structure, since bioarchaeological studies offer enormous possibilities for explaining the populations of the past and, in particular, their demographic regime. This work analyses different paleodemographic proposals of collective graves or funerary ensemble from Recent Prehistory, perhaps the contexts in which this type of study encounters the greatest difficulties. The aim is to highlight and compare the methodological and interpretative solutions proposed for the selected studies. In any case, it is evident that demographic analysis applied to collective burials in Recent Prehistory offers a better comprehensive understanding of these mortuary spaces as well as the possibility of confronting comparisons based on the unity of method.
\end{abstract}

Keywords: paleodemography; Prehistory; collective tombs; Iberian Peninsula

\title{
INTRODUCCIÓN
}

La investigación de la demografía ocupa un puesto importante en el estudio de las comunidades humanas de cualquier época, pues atañe a la interacción entre aspectos biológicos, sociales y económicos cuyo interés es evidente. Su importancia, en consecuencia, no varía cuando se trata de estudiar la demografía de poblaciones prehistóricas, en este caso a través de la Arqueología y de la Antropología Física, aunque las dificultades aumentan al partirse de unas fuentes tan limitadas como son los restos funerarios de la época de los cuales solo se maneja una reducida muestra de la población original. Y, siendo el interés el mismo, los problemas pueden llegar a ser particularmente arduos cuando el objeto de estudio son los osarios de los sepulcros colectivos de la Prehistoria Reciente, pues el carácter acumulativo y el desorden frecuente que reina en este tipo de tumbas suponen una traba adicional para los estudios antropológicos previos a los análisis propiamente demográficos.

A este primer inconveniente han de sumarse las limitaciones propias de la disciplina paleodemográfica, que en las últimas décadas han sido recurrentemente objeto de debate. El principal problema en los estudios de sociedades prehistóricas es el de la representatividad de las muestras pues, mientras la paleodemografía requiere un estudio cuantitativo exacto de una determinada población en un lugar y tiempo concretos, la arqueología solo cuenta para su estudio con colecciones incompletas, para nada representativas de toda la población original.

Las dificultades a la hora de reconstruir el perfil demográfico de una población prehistórica no han impedido, sin embargo, que se hayan producido avances notables en la materia. Muchos han sido los estudios que se han realizado con éxito sobre poblaciones antiguas a partir de colecciones arqueológicas, bien centrados en parámetros tales como el tamaño de la 
población, $\mathrm{o}$ en las transiciones demográficas entre distintos periodos prehistóricos, especialmente aquellos dedicados a la investigación de la transición neolítica (Bocquet-Appel, 2002).

Generalmente estos trabajos buscaban reconstruir perfiles demográficos teóricos con el fin de comparar y medir su tamaño en el tiempo y en el espacio (Ortega, 2004; Séguy y Buchet, 2013). En esta línea podrían destacarse, entre otros, los clásicos estudios de G. Acsádi y J. Nemeskèri (1970) sobre poblaciones calcolíticas húngaras, en los que se aplicó por primera vez la metodología que fundamentaría la investigación paleodemográfica. Poco más adelante, se desarrollaron otra serie de estudios de indudable repercusión en este campo, como el de D. Brothwell (1972) sobre población arqueológica en Gran Bretaña o los bien conocidos de la llamada "escuela francesa” (Masset, 1973; Bocquet-Appel y Masset, 1977; 1982). También merece ser mencionada la investigación de S. Welinder (1979) sobre las transiciones poblacionales prehistóricas suecas mediante el estudio de diversas colecciones antropológicas.

Pero más allá del simple interés de conocer la demografía de las poblaciones del pasado, muchos investigadores han encontrado en la paleodemografía una herramienta de enorme utilidad para desentrañar ciertas pautas culturales de las fórmulas funerarias, sobre todo en lo que respecta a la presencia en los contextos mortuorios de determinados grupos de edad o sexo. Precisamente en esta línea se sitúa un buen número de investigaciones en la península ibérica, en especial de aquellas que abordan el estudio de enterramientos colectivos con numerosos individuos pertenecientes a distintas clases de edad (Fernández-Crespo y De La Rúa, 2016; Silva, 2003; Waterman y Thomas, 2011). Y fruto de ello, pese a las dificultades que conlleva el análisis antropológico de los sepulcros colectivos, es el nacimiento de una línea de investigación específica sobre los osarios colectivos propios de tales yacimientos. Desde esta perspectiva, en el presente trabajo se revisan y analizan una serie de estudios sobre el perfil demográfico deducido del estudio de determinados enterramientos colectivos prehistóricos con el fin de conocer sus posibilidades interpretativas, así como de hacer algunas puntualizaciones sobre la metodología más comúnmente utilizada en los trabajos de paleodemografía prehistórica de la Península Ibérica.

\section{PosibILIDAdES Y LiMITACIONES EN LOS ESTUDIOS PALEODEMOGRÁFICOS}

\section{1. Limitaciones metodológicas y muestrales}

El principal problema al que se enfrenta la investigación de las poblaciones prehistóricas es la representatividad demográfica de las series esqueléticas, especialmente aquellas procedentes de sepulcros colectivos. Numerosos autores 
han cuestionado tradicionalmente que dichas muestras se hallen completas y sean representativas de la población original (Angel, 1969; Masset, 1986; Ubelaker, 1974). Esa falta de correspondencia entre la tasa de mortalidad real de una población y los restos humanos que se recuperan en las excavaciones puede ser debida bien a una conservación diferencial motivada por diversos fenómenos tafonómicos o bien a elementos culturales en el patrón de selección de los individuos en un enterramiento. Pese a que cualquier tipo de cementerio constituye una pequeña porción del total de muertes que se producen en una población determinada, cuando hablamos de sepulcros colectivos en la Prehistoria este fenómeno se multiplica, ya que los cadáveres presentan mayores problemas de conservación, pero además porque se desconoce en gran medida qué pautas de enterramiento se siguieron para darles sepulcro a estos individuos.

Por otro lado, la dificultad para estimar con exactitud la edad de muerte del individuo tampoco favorece el análisis poblacional, especialmente en los adultos, para los que se manejan rangos de edad excesivamente amplios e imprecisos (Bocquet-Appel y Masset, 1982). Recordemos que se trata de un problema agravado especialmente en los osarios prehistóricos debido a la mezcla y fragmentación de los restos, así como a la escasez de conexiones anatómicas, que en muchas ocasiones se reducen a uno o dos individuos correspondientes a enterramientos intrusivos o a fases de enterramiento más modernas (Delibes, 2010; Rojo et alii, 2005). Por esta razón, el análisis antropológico en los sepulcros colectivos resulta mucho más complicado y conlleva estimaciones de edad y sexo muy vagas, dificultando también el estudio de los parámetros poblacionales. Se trata de un problema que provoca cierta desesperación en el desarrollo de estos estudios, ya que cuánto más grande sea esta desviación del intervalo de edad del individuo -o fragmento de individuo, tal y como aparecen los restos humanos en la mayoría de colecciones procedentes de sepulcros colectivos-, menor será la información demográfica que se obtenga (Jiménez Brobeil et alii, 1991).

Por si fuera poco, también se cuestiona la fiabilidad en las estimaciones de sexo y edad, al basarse fundamentalmente en poblaciones contemporáneas cuyo desarrollo biológico no tiene por qué asimilarse al de la muestra arqueológica que se estudia. De hecho, Bocquet-Appel y Masset (1982) observaron una falta de correspondencia considerable entre las estimaciones de la edad cronológica y la edad biológica ${ }^{1}$ de la muerte de un individuo, lo cual supone un desajuste intrínseco en la estimación de edad, especialmente en adultos. Otros investigadores, como J.W. Wood et alii (1992) advierten además sobre la dificultad que supone estimar el riesgo real de muerte en un determinado grupo

\footnotetext{
${ }^{1}$ Se trata de la edad que se calcula desde el nacimiento de un individuo, mientras que la edad biológica se corresponde con el proceso de osificación que se observa en los huesos.
}

BSAA arqueología, LXXXIV, 2018, pp. 71-93.

E-ISSN: 2530-6367 
de edad "arqueológico" puesto que existen circunstancias diversas que no siempre se ven reflejadas en los restos óseos. Estos mismos autores afirman también que los cambios en la fertilidad de una población podían causar desajustes mayores que la propia mortalidad en los perfiles demográficos arqueológicos reconstruidos a partir de la distribución de las edades de muerte de los individuos, por lo que las curvas de mortalidad en muchos casos podrían reflejar en realidad picos de fertilidad enmascarados. Otros estudios posteriores en poblaciones históricas han demostrado igualmente que los valores demográficos procedentes de censos parroquiales no siempre se corresponden con los obtenidos mediante el estudio de los restos humanos que se recuperan del cementerio correspondiente (Ortega, 2004; Séguy y Buchet, 2013), haciendo más difícil aun confiar en los avances del estudio demográfico de poblaciones antiguas.

A pesar de todo, resulta esperanzador que las dificultades metodológicas a las que se ha enfrentado esta disciplina, lejos de paralizar la investigación, han servido de impulso para desarrollar nuevos métodos capaces de corregir las anomalías de representación poblacional propias de una muestra arqueológica. De esta forma es posible establecer qué tipo de población se trabaja o inferir a qué pueden deberse la ausencia o presencia de ciertos grupos de edad. Por ejemplo, Bello y Andrews (2006) señalan que una mayor representatividad de adultos en muestras arqueológicas puede deberse a un fenómeno de conservación diferencial, por lo que los contextos colectivos, en los que aparecen huesos mezclados y fragmentados, estos problemas han de estar bien presentes (Campillo 1995: 319). De hecho, pese a que la escasa representación de subadultos en contextos funerarios prehistóricos nunca se corresponde con los altos índices de mortalidad infantil que cabrían esperar en sociedades preindustriales (Ledermann, 1969), se empieza a considerar que la presencia de individuos inmaduros en sepulcros colectivos de la Prehistoria reciente no es para nada desdeñable, puesto que su representación se acerca, en muchos casos, al 30\% del total de la muestra (Beck, 2016; Waterman y Thomas, 2011). Efectivamente, ciertas investigaciones advierten sobre el sesgo metodológico que puede existir a la hora de analizar según qué contextos funerarios, sobre todo aquellos yacimientos que parecen estar destinados a un determinado segmento de la población.

También puede ocurrir que el sesgo de selección se deba al tamaño de la muestra, la cual sea tan pequeña que altere considerablemente la curva demográfica que se obtiene. Tanto es así que el número de individuos mínimo que ha de constituir un conjunto esquelético para poder realizar cálculos paleodemográficos se establece en 50 (Bocquet-Appel y De Miguel, 2002: 32; Etxeberria y Herrasti, 2007:195), aunque otros autores lo elevan a 100 para que los resultados sean estadísticamente consistentes (Séguy y Buchet, 2013). Este último requisito supone una traba adicional en el estudio de sepulcros colectivos 
especialmente megalíticos, puesto que aquellos en los que se sobrepasa el centenar de sujetos enterrados constituyen verdaderas excepciones.

Por último, los cementerios suelen reutilizarse a lo largo del tiempo, bien sea por una misma comunidad o por varias en épocas distintas. Precisamente, este sería el caso paradigmático de las tumbas colectivas prehistóricas, cuyos parámetros demográficos pueden verse considerablemente alterados por la deposición de diferentes individuos durante diversos ciclos de enterramiento, por lo que la temporalidad del depósito ha de tenerse en cuenta en el estudio. Así lo demuestran los innumerables testimonios de reutilización funeraria de dólmenes que albergan población del Neolítico Final, donde tanto los materiales campaniformes como la datación absoluta de algunos individuos manifiestan que los fenómenos de reutilización dolménica fueron comunes en comunidades calcolíticas e incluso posteriores (Aranda Jiménez et alii, 2017; FernándezEraso y Mujika, 2013). Por lo tanto, la naturaleza acumulativa de este tipo de contextos funerarios hace que algunos autores desaconsejen su estudio paleodemográfico y aún más que se tomen como reflejo real de la población original, puesto que probablemente la muestra esté compuesta por individuos de poblaciones diferentes (Bocquet-Appel y De Miguel, 2002).

En resumen, las numerosas dificultades que atañe la revisión de una población arqueológica cuestionan la propia disciplina paleodemográfica. Sin embargo, en ausencia de fuentes escritas que puedan aportar datos más fiables, la posibilidad de comprender cómo eran las poblaciones pretéritas se reduce principalmente a los restos humanos. Por esta razón, los estudios poblacionales han de abordarse con máxima cautela desde el momento en el que se inicia la investigación, siendo fundamental proceder con un sistema de excavación sistemático que permita registrar todos los datos y observaciones antropológicas in situ para evitar confusiones e interpretaciones erróneas en los análisis posteriores (Masset, 1986; Ubelaker, 1974). De esta manera, la paleodemografía se ofrece como una disciplina capaz de aproximarse a la estructura poblacional de las sociedades prehistóricas (Hassan, 1978), siempre y cuando afronte los problemas derivados de la falta de representatividad en las muestras arqueológicas, dando lugar a una serie de principios teóricos que han de fundamentar cualquier estudio paleodemográfico.

\section{2. Fundamentos teóricos para la reconstrucción paleodemográfica}

El estudio de poblaciones antiguas a partir del análisis de restos óseos comenzó en la primera mitad del siglo XX, momento en el que las colecciones osteológicas se empiezan a considerar fuentes de estudio válidas para comprender las dinámicas de los grupos humanos. El desarrollo de técnicas capaces de generar modelos representativos mediante el estudio antropológico 
de los huesos se debe sobre todo a la elaboración de tablas de vida y de mortalidad, muy utilizadas en estudios demográficos contemporáneos. Se trata de un método ampliamente abordado en la literatura paleodemográfica y ha resultado ser idóneo para soslayar los peligros que conlleva asimilar el número de individuos de la muestra esquelética con el comportamiento demográfico real de una población en concreto (Roca, 2012). La aplicación de este método en colecciones arqueológicas fue propuesta por G. Acsàdi y J. Nemèskeri en 1970 a partir de las tablas elaboradas por la ONU en 1958, las cuales permiten el realizar el cálculo de parámetros demográficos (probabilidad de fallecimientos por edad, esperanza de vida al nacer, supervivencia, etc.) y observar su mayor o menor ajuste al esquema de mortalidad arcaico. Más tarde, en 1973, K. Weiss y H. Martin retomaron los modelos de reconstrucción poblacional, convirtiéndose igualmente en estudios de referencia para el desarrollo de la investigación en este campo.

De esta forma se consiguió minimizar con relativo éxito aquellas alteraciones demográficas derivadas de determinados patrones funerarios o de problemas en la conservación de la muestra esquelética (Hassan, 1978; Welinder, 1979). Además, fue posible estimar la mortalidad y supervivencia teóricas en estas poblaciones mediante el análisis de la distribución por grupos de edad de los individuos identificados en el yacimiento. De este modo, se logró reconstruir de forma indirecta los patrones de mortalidad, fertilidad y crecimiento vegetativo de un grupo humano a partir del estudio de la población arqueológica y no mediante la observación directa de su comportamiento demográfico, algo que trascendió notablemente en la investigación prehistórica.

Para llevar a cabo este objetivo, la disciplina paleodemográfica necesita partir del principio de uniformidad biológica según la cual se reconstruyan y analicen los parámetros poblacionales, asumiendo que los límites biológicos de las tasas de mortalidad y fecundidad del ser humano permanecen inalterables bajo las mismas condiciones ecológicas (Howell, 1976). Dicha premisa sustenta la base de la teoría paleodemográfica ya que asume de forma hipotética que el comportamiento de las comunidades del pasado permanece inalterable con el tiempo. Por lo tanto, el modelo de población arcaica en el que se basan estos estudios se aproxima de manera natural a una situación de estabilidad, es decir, que su tasa de crecimiento vegetativo tiende a cero. Esta asunción lleva a aceptar además un principio de estacionalidad, entendiendo que la tasa de crecimiento de la población es nula, que existe un reparto normal de los segmentos poblacionales y que no se producen aportes foráneos significativos (Acsádi y Nemeskéri, 1970; Weiss y Martin, 1973).

Aunque todas estas premisas se basen en conceptos demasiado teóricos y probablemente alejados de la realidad prehistórica, la hipótesis de población estacionaria -o estable- y cerrada, se suele aplicar como modelo demográfico ya 
que muchos autores consideran que el crecimiento poblacional arcaico se caracteriza principalmente por un ritmo lento y estable (Chamberlain, 2009; Howell, 1976; Weiss y Martin, 1973). Así lo establece la denominada hipótesis Halley, mediante la que se asume que las poblaciones tienden a un equilibrio natural con un crecimiento vegetativo nulo (Acsádi y Nemeskéri, 1970: 61), y gracias a la cual es posible reconstruir perfiles demográficos teóricos, tomando como referencia el esquema de mortalidad arcaico establecido por S. Ledermann en 1969. Dicho modelo descansa en el estudio de diversas poblaciones preindustriales caracterizadas por unas altas tasas de mortalidad infantil y una baja esperanza de vida, atribuibles ambas a sociedades arcaicas (Acsádi y Nemeskéri, 1970; Angel, 1969; Chamberlain, 2009; Howell, 1976; Weiss y Martin, 1973). También existen otros modelos demográficos reconstruidos a partir de poblaciones preindustriales, aunque el propuesto por Ledermann (1969) suele escogerse en la mayoría de los casos por la gran variedad de modelos demográficos que ofrece (Alesan et alii, 1999: 289)

Por consiguiente, la aceptación de la premisa de uniformidad biológica es fundamental a la hora de avanzar en el análisis paleodemográfico, siempre que la interpretación de la mortalidad real de una población antigua a partir del registro arqueológico se realice con cautela. De hecho, el planteamiento teórico del que parte la paleodemografía también ha sido fuertemente criticado al fundamentarse en unas premisas quizás demasiado limitadas como para llegar a comprender las dinámicas de las poblacionales en el pasado. Lo cierto es que estos modelos no tienen en cuenta las posibles catástrofes demográficas o los movimientos de población que indudablemente alterarían la composición poblacional de las comunidades prehistóricas. Pese a los grandes avances en investigación genética acerca de los movimientos migratorios en la Prehistoria que en los últimos años están ganando importancia en el campo de la demografía prehistórica (Olalde et alii, 2018; Szécényi-Nagy et alii, 2017), sigue siendo difícil determinar cómo era el comportamiento de las poblaciones en determinadas épocas y áreas geográficas. Además, algunos autores señalan que el desconocimiento documental de las comunidades prehistóricas obliga a asumir hipótesis únicamente basadas en la comparación etnográfica y en el estudio de la evolución demográfica a lo largo de la historia, por lo que es probable que se reproduzcan modelos poco representativos de las poblaciones prehistóricas (Bocquet-Appel y Masset, 1982).

No obstante, desde otros trabajos sí se defiende la utilidad de los métodos paleodemográficos aplicados al estudio de osarios prehistóricos, siempre que se haga con el objetivo de detectar patrones o anomalías en la composición poblacional en este tipo de enterramientos. La alteración de los índices de mortalidad, supervivencia o la esperanza de vida al nacer en determinados grupos de edad con respecto a los valores que se pueden esperar de una población arcaica puede aportar información valiosa sobre qué grupo tiene 
acceso preferencial a estas tumbas (Fernández-Crespo y De la Rúa, 2016). Por esta razón, en muchas ocasiones la aplicación de los métodos demográficos se ha orientado preferiblemente hacia la búsqueda de estos patrones demográficos y no tanto a establecer una estimación directa de la estructura de la población, lo cual ha permitido una mejor interpretación del sepulcro.

\subsection{Soluciones metodológicas comúnmente adoptadas en los yacimientos de enterramiento colectivo}

La mayor parte de trabajos en los que se analizan los segmentos de población de un sepulcro colectivo, o conjunto de sepulcros prehistóricos, se apoyan en los fundamentos teóricos paleodemográficos para solucionar los graves problemas de representación propios de estos enterramientos. Las soluciones metodológicas más utilizadas en los trabajos de demografía prehistórica se basan en la construcción de las ya mencionadas tablas de mortalidad. Ahora bien, las críticas que ha sufrido este método lo largo de la investigación paleodemográfica han propiciado que buena parte de estos estudios hayan contemplado la utilización una serie de factores de corrección que minimizan las anomalías de representatividad de las muestras. En este sentido, Bocquet-Appel y Masset (1977, 1982), enfatizan la necesidad de aplicar el Índice de Juventud entre los estimadores paleodemográficos propuestos para poblaciones arqueológicas, mediante los cuales se corrigen o disminuyen los errores de representación de determinados segmentos de la población que no son fáciles de determinar por razones de conservación o por la dificultad de diagnosticar la edad de los restos humanos.

El Índice de Juventud (IJ) ${ }^{2}$ establece la relación entre adultos e inmaduros y resulta un indicador esencial a la hora de detectar anomalías por la excesiva infrarrepresentación de individuos menores de 20 años en poblaciones arqueológicas. Además, las diferencias morfológicas del esqueleto subadulto respecto al adulto, hacen más identificable este grupo de edad en las muestras arqueológicas, por lo que el índice de juventud está considerado como un buen estimador paleodemográfico en poblaciones antiguas (Bocquet-Appel y Masset, 1982; Fernández-Crespo y De La Rúa, 2016). No obstante, aunque este índice excluye a los individuos menores de 5 años, la presencia de éstos en los

\footnotetext{
${ }^{2}$ Se obtiene al dividir el número de sujetos fallecidos entre los 5 y 14 años por el número de fallecidos mayores de 20 años y se expresa mediante la ecuación: $D_{5-14} / D_{20-\infty}$. Proporciona un buen índice de estimación de individuos infantiles sin tener en cuenta los datos brutos de los sujetos menores de 5 años (Sellier, 1996: 195). También son útiles para el estudio paleodemográfico las diferentes proporciones que se obtienen entre los grupos quinquenales de individuos subadultos, precisamente por la mayor precisión con la que se puede estimar la edad de muerte del individuo (Bocquet-Appel y Masset, 1977).
} 
yacimiento ha de valorarse prestando especial atención a los problemas de conservación de los infantiles como consecuencia de la escasa mineralización y débil estructura de los huesos inmaduros (Bello y Andrews, 2006). Por consiguiente, una excavación exhaustiva y un registro sistemático de todos los elementos óseos proporcionaría cierta seguridad a la hora de contabilizar los sujetos infantiles, ya que hay elementos como los dientes o las diáfisis de los huesos largos que presentan condiciones óptimas de preservación al mineralizarse en etapas muy tempranas del desarrollo (Sellier, 1996). Dicha sistematización posibilita analizar las anomalías de representación en individuos subadultos teniendo cierta convicción de que la falta de estos sujetos no se debe a procesos tafonómicos que hayan eliminado las osamentas de los individuos más jóvenes. Dicha sistematización se torna verdaderamente importante en los enterramientos colectivos, ya que la mezcla y fragmentación de los elementos óseos puede alterar los parámetros de representación poblacional.

\section{APORTACIÓN DE ALGUNOS ESTUdIOS PENINSULARES A LA METODOLOGÍA PALEODEMOGRÁFICA}

\section{1. Estudios seleccionados}

En esta revisión se ha seleccionado un conjunto de estudios realizados en la Península Ibérica sobre sepulcros colectivos prehistóricos. Se han elegido aquellos más representativos según el tipo de análisis demográfico, la etapa prehistórica estudiada y las limitaciones en la representación de las muestras esqueléticas. A continuación se presentan los ejemplos escogidos, así como el método empleado y las conclusiones derivadas de cada uno de ellos. Se tiene en cuenta también el área geográfica, la época y el tipo de estudio (interpretativo o comparativo):

TABLA I. Estudios paleodemográficos analizados en este trabajo. TM*: Tablas de mortalidad (Acsàdi y Neméskeri, 1970); IJ**= Índice de Juventud (Bocquet-Appel y Masset, 1977)

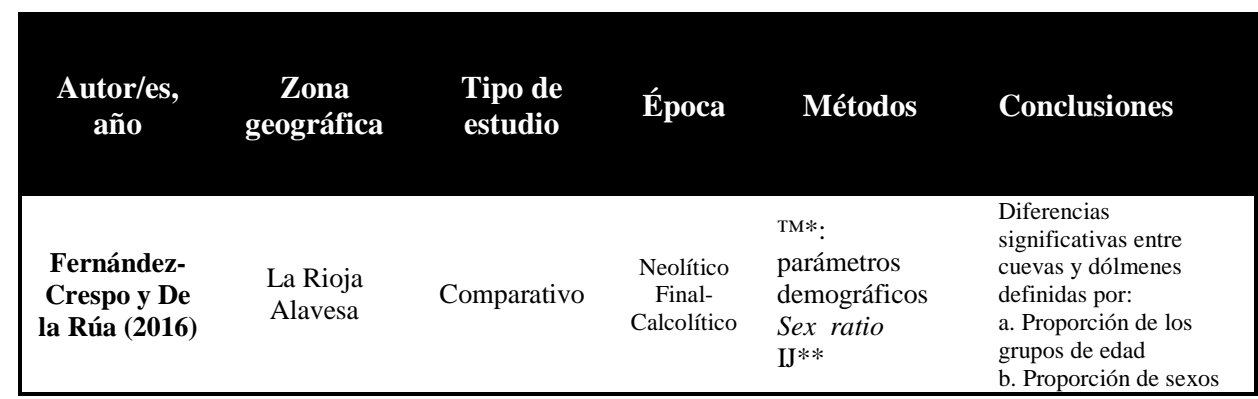

BSAA arqueología, LXXXIV, 2018, pp. 71-93.

E-ISSN: 2530-6367 


\begin{tabular}{|c|c|c|c|c|c|}
\hline $\begin{array}{c}\text { Etxeberria } \\
\text { y Herrasti } \\
(2007)\end{array}$ & $\begin{array}{l}\text { La Rioja } \\
\text { Alavesa }\end{array}$ & Interpretativo & $\begin{array}{l}\text { Neolítico } \\
\text { Final- } \\
\text { Calcolítico }\end{array}$ & $\begin{array}{l}\text { TM*: } \\
\text { parámetros } \\
\text { demográficos } \\
\mathrm{IJ}^{* *}\end{array}$ & $\begin{array}{l}\text { a. Infrarrepresentación } \\
\text { de individuos menores } \\
\text { de } 5 \text { años, en contraste } \\
\text { con la representación } \\
\text { de subadultos de 5-14 } \\
\text { años } \\
\text { b. "posibilidad de un } \\
\text { perfil de población de } \\
\text { tipo catastrófico" }\end{array}$ \\
\hline $\begin{array}{c}\text { Domínguez } \\
\text { del Triunfo } \\
\text { (2013) }\end{array}$ & $\begin{array}{l}\text { Mediterráneo } \\
\text { Oriental y } \\
\text { Occidental } \\
\text { (península } \\
\text { ibérica) }\end{array}$ & Comparativo & $\begin{array}{l}\text { Edad del } \\
\text { Bronce }\end{array}$ & $\begin{array}{l}\text { TM*: } \\
\text { parámetros } \\
\text { demográficos } \\
\text { IJ** } \\
\text { Sex ratio }\end{array}$ & $\begin{array}{l}\text { a. No se destacan } \\
\text { importantes } \\
\text { anomalías } \\
\text { demográficas. } \\
\text { b. Comparativa: } \\
\text { diferentes proporciones } \\
\text { de subadultos entre el } \\
\text { Mediterráneo Oriental y } \\
\text { el Mediterráneo } \\
\text { Occidental } \\
\end{array}$ \\
\hline $\begin{array}{l}\text { Jiménez et } \\
\text { alii (1991) }\end{array}$ & $\begin{array}{l}\text { Andalucía } \\
\text { Oriental }\end{array}$ & Interpretativo & $\begin{array}{l}\text { Neolítico } \\
\text { Calcolítico } \\
\text { y Edad del } \\
\text { Bronce }\end{array}$ & $\begin{array}{l}\text { TM*: parámetros } \\
\text { demográficos }\end{array}$ & $\begin{array}{l}\text { a. Muestra válida para } \\
\text { la Edad del Bronce } \\
\text { - Mortalidad alta en la } \\
\text { primera infancia } \\
\text { - Ligero predominio de } \\
\text { mujeres }\end{array}$ \\
\hline
\end{tabular}

\subsection{Descripción de los métodos paleodemográficos utilizados en los estudios analizados}

En uno de los estudios más recientes, T. Fernández-Crespo y C. De la Rúa (2016) comparan los patrones de enterramiento en cuevas y en contextos megalíticos de la Rioja Alavesa entre el Neolítico Final y el Calcolítico Inicial, (IV-III milenio cal. BC). En total se estudian 261 individuos procedentes de tres abrigos rocosos o cuevas del Valle del Ebro, entre los que se encuentran Las Yurdinas II, Peña Larga y La Peña de Marañón; y de tres dólmenes de la misma región, concretamente, Alto de La Huesera, San Martín y Peña Guerra II (Fernández-Crespo y De La Rúa, 2016).

Todas las muestras seleccionadas se analizaron bajo los mismos criterios metodológicos en cuanto a la estimación de edad y sexo con lo que se minimiza el error inter-observador en el estudio (Ibídem: 289). Se trata de un problema del que adolecen algunos trabajos paleodemográficos en los que se agrupan colecciones estudiadas bajo diferentes criterios metodológicos, sin tener en cuenta la distribución por grupos de edad quinquenal, necesaria para calcular los parámetros demográficos. No obstante, se debe tener en cuenta que la distribución quinquenal pocas veces es viable a partir del estudio de los huesos, principalmente debido a las grandes desviaciones en la estimación de edad de 
los adultos y por el hecho de que muchos individuos pueden encontrarse entre dos intervalos quinquenales.

T. Fernández-Crespo y De la Rúa solucionan este problema mediante un reparto proporcional del total de los individuos no asignados a los quinquenios mediante el "principio de minimización de anomalías demográficas" o "de conformidad" propuesto por P. Sellier (1996). Por medio de este principio se elige el reparto de individuos que más se ajuste a los índices paleodemográficos formulados por Bocquet-Appel y Masset en 1977, atendiendo especialmente al factor de corrección determinado por la proporción de individuos subadultos (IJ). Además, como ya se ha mencionado, el estimador IJ proporciona información sumamente valiosa desde el punto de vista demográfico, puesto que permite ajustar la esperanza de vida al nacer de la población que se estudia, posiblemente alterada por la escasez de infantiles ${ }^{3}$.

Estos factores de corrección consiguen reducir las anomalías demográficas que surgen de una imprecisa estimación de edad. En el trabajo de T. FernándezCrespo y C. De la Rúa estas correcciones se emplean en las propias tablas de mortalidad, camuflando de esta forma la ausencia de individuos menores de 5 años, un fenómeno que, como veremos, se repite en un buen número de colecciones arqueológicas. Con ello se consigue además un reparto más proporcional de los fallecidos pertenecientes a edad adulta. De esta manera es posible trazar perfiles de mortalidad, supervivencia, esperanza de vida, etc., más acordes a los valores teóricos de Ledermann (1969). Una vez aplicados los factores de corrección, las anomalías de representación demográfica pueden ser analizadas con mayor fiabilidad, especialmente entre los más jóvenes (Fernández-Crespo y De La Rúa, 2016: 289).

Los resultados del análisis en el estudio de T. Fernández-Crespo y C. De La Rúa (2016) revelan un patrón característico en el que la incorporación de mujeres, infantiles y adultos en general presenta diferencias estadísticamente significativas entre cuevas y dólmenes. Sin duda, esta cuestión vendría a corroborar la hipótesis de reclutamientos selectivos en los sepulcros megalíticos vinculados quizás a ciertos privilegios sociales (Delibes de Castro, 1995; Guerra et alii, 2009; Masset, 1986). Resulta curioso puesto que no sucede lo mismo en las cuevas analizadas, en las que es más frecuente registrar la presencia de grupos subadultos y una representación de sexos más acorde con la tendencia

\footnotetext{
${ }^{3}$ La esperanza de vida al nacer $\left(\mathrm{e}_{0}{ }_{0}\right)$ se sitúa en torno a los 25-30 años para poblaciones dependientes de un tipo de economía de subsistencia basada en la agricultura y ganadería, es decir, para comunidades de Prehistoria Reciente en las que se ha completado el proceso de sedentarización. Ahora bien, uno de los problemas fundamentales que se derivan de la ausencia de individuos infantiles y perinatales en los sepulcros colectivos prehistóricos hace que la esperanza de vida al nacer se dispare hasta casi los 50 años (Sellier, 1996), ya que la mayor parte de fallecidos se concentran en edades avanzadas, desvirtuando por completo el probable modelo de mortalidad de la población estudiada.
} 
natural de proporción 1:1 entre mujeres y hombre existente en las sociedades humanas.

En el caso de los sepulcros megalíticos, este fenómeno probablemente obedezca, como decimos, a las pautas de reclutamiento selectivo que ya observó Masset (1986) en los dólmenes franceses, precisamente en uno de los trabajos pioneros sobre paleodemografía en este tipo de contextos, en los que parecía registrarse un predominio de adultos bastante discordante con el tipo de mortalidad arcaica. Por lo tanto, este estudio vendría a corroborar de forma empírica estas pautas de selección. Aunque la corrección de los parámetros demográficos consiga suavizar las grandes desproporciones que observaban $a$ priori, la infrarrepresentación de infantiles y mujeres es perfectamente observable. Aquellos individuos que se encuentran en la franja de edad correspondiente con la primera infancia (0-5) son prácticamente ausentes, algo que, descartados los ya comentados problemas de conservación y registro, sí podría indicar la exclusión de estos grupos de edad como reflejo de un estatus social aun no alcanzado, relegando quizás su tratamiento funerario a la esfera privada (Waterman y Thomas, 2011: 171; Sellier, 1996).

Sin alejarnos de la comarca de la Rioja Alavesa, conviene detenerse también en el estudio paleodemográfico del abrigo rocoso de San Juan ante Portam Latinam realizado por F. Etxeberria y L. Herrasti (2007). El enterramiento, datado en 3338-3095 cal B.C. (Armendáriz, 2007) cuenta con un número mínimo de individuos establecido en 338, es decir, una muestra suficientemente grande para llevar un cabo un análisis demográfico. En ese caso se realiza una distribución de edad por quinquenios, que se soluciona repartiendo la parte proporcional correspondiente de los individuos indeterminados de cada grupo etario de acuerdo con el número de individuos de edad sí determinada (Etxeberria y Herrasti, 2007). Probablemente los autores optan por esta corrección en el reparto de edades debido al gran tamaño de la muestra y a la representación óptima de todos los grupos de edad.

El resultado de los parámetros demográficos en individuos subadultos y el Índice de Juventud nuevamente no se corresponden con el tipo de mortalidad arcaica de S. Ledermann (1969). También detectan una anomalía importante en la representación de menores de 5 años, sin embargo, la proporción entre los grupos de edad 5-9 años y 10-14 supera el índice esperado. Por consiguiente, los autores confirman una auténtica inversión de la curva de mortalidad infantil marcada por una considerable infrarrepresentación de individuos menores de un año pero con una mayor presencia de subadultos mayores de cinco años de la que se constata en los sepulcros megalíticos. De esta forma, sugieren que la "probabilidad de un perfil de población catastrófico ha de ser tenida en cuenta" (Etxeberria y Herrasti, 2007: 198), asociada en este caso además con evidencias de violencia presentes en un buen número de individuos encontrados en este 
hipogeo, aunque no descartan que el patrón de enterramiento obedezca a algún tipo de pauta intencional.

Cabe destacar que el análisis de los valores demográficos de San Juan ante Portam Latinam evidencia que difieren considerablemente de la mayoría de los enterramientos colectivos entre el Neolítico Final y Calcolítico (Etxeberria y Herrasti, 2007). Hay que considerar que la alteración de los datos poblacionales bajo el análisis de la temporalidad de las secuencias funerarias demuestra que el carácter acumulativo de las tumbas colectivas provoca sesgos de representación en grupos de edad y sexo, por lo que la variable cronológica debería tenerse muy en cuenta a la hora de interpretar la composición poblacional de sepulcros de este tipo, especialmente si no hay registro o constancia de diferentes niveles de ocupación. Ejemplo de ello, es un estudio reciente en el que se combinan los datos demográficos con el análisis cronológico que permite interpretar la secuencia funeraria como un enterramiento en dos fases, una de las cuales podría estar relacionada con episodios de conflictividad y violencia (FernándezCrespo et alii., 2018). Por lo tanto, parece clara la necesidad de entender mejor las cronologías funerarias para interpretar y comprender el funcionamiento de los sepulcros colectivos prehistóricos, no solo desde el punto de vista temporal sino también desde la dimensión social de la actividad funeraria.

Otro trabajo de interés es el realizado por Sylvia A. Jiménez Brobeil, B. Robledo y F. Coronado Ortiz, (1991) en la zona de Andalucía Oriental. La muestra recogida procede de yacimientos (cuevas, covachas y dólmenes), correspondientes al Neolítico, Edad del Cobre y Edad del Bronce. El estudio se ha planteado teniendo en cuenta la problemática implícita en los análisis paleodemográficos. No obstante, en los tres periodos se usa una metodología uniforme para determinar sexo y edad. Al contrario que en los otros estudios mencionados, los individuos son clasificados según los grupos de edad clásicos en Antropología Física, es decir, Infantil I, Infantil II, Juvenil, Adulto, Maduro y Senil, por lo que en este caso el estudio se tornaría más descriptivo, no sin restar el valor informativo que proporciona conocer la composición demográfica de la población hallada en el yacimiento.

En cuanto a los parámetros demográficos, los autores proponen que, según las tablas de vida -infantiles y adultos-, los datos más fiables para hacer una aproximación paleodemográfica pertenecen a la Edad del Bronce. Los autores sostienen que este fenómeno pueda deberse a que los individuos proceden de inhumaciones individuales y de yacimientos excavados con técnicas modernas. En cuanto a la distribución por sexos de todos los períodos se documenta curiosamente una mayor mortalidad de las mujeres respecto a los hombres en el intervalo de edad de 21-40 años, probablemente como consecuencia de los riesgos de la maternidad. No obstante, en la Edad del Cobre y la Edad del Bronce la diferencia es prácticamente mínima. Esta situación contrastaría con 
los enterramientos colectivos riojanos de tipo dolménico, en los que es común registrar una sobrerrepresentación masculina importante.

Por último, apelando a estudios más extensos, se puede mencionar el trabajo de H. Domínguez del Triunfo (2013). Se trata de un análisis comparativo para yacimientos del contexto mediterráneo del II milenio a.C., entre los que destacan un importante número de yacimientos peninsulares de la Edad de Bronce que se comparan con los del extremo oriental del Mediterráneo. La autora expone con gran acierto una limitación inicial de gran calibre para la aplicación de técnicas paleodemográficas que responde a una falta de homogeneidad metodológica en la distribución de los sujetos en los grupos de edad (Ibídem.: 14). Para solucionar la falta de correspondencia entre las categorías de edad clásica y quinquenal compara las proporciones entre individuos adultos y subadultos de forma genérica, aunque también opta por la agrupación de los estudios en función de esta variable, lo que le permite la comparación parcial de los estudios (Domínguez del Triunfo, 2013: 20-21). Esta solución metodológica resulta útil para el cálculo de los parámetros demográficos e índices de juventud. Los resultados, en este caso, no se desvían demasiado del modelo de mortalidad arcaica tanto en el área del Mediterráneo oriental como en el del occidental. No obstante, también se aprecian algunas excepciones que impiden hablar de un patrón de enterramiento homogéneo, pero que la autora atribuye a fenómenos de preservación diferencial.

Otro aspecto importante que proporcionan los parámetros demográficos analizados en la zona occidental del Mediterráneo es que la proporción media entre sexo femenino y masculino responde a una distribución normal con un ratio se sitúa en torno al 50\% (Domínguez del Triunfo, 2013: 22). En los casos en los que no se cumple la proporcionalidad entre hombres y mujeres, se tienen en cuenta factores como el mayor riesgo de muerte en mujeres en edades fértiles, las posibles diferencias socio-económicas posiblemente reflejadas en los enterramientos, además de los problemas de conservación de la muestra. Si se consideran las limitaciones metodológicas de este estudio, podría decirse que existe mayor adecuación al esquema de mortalidad natural durante la Edad del Bronce en los territorios mediterráneos, sin que se pueda inferir un patrón de exclusión en los cementerios tan claro como en otros trabajos.

\section{DISCUSIÓN}

La literatura paleodemográfica ya advierte de la dificultad que supone reconstruir perfiles demográficos a partir de muestras arqueológicas. Si bien se ha demostrado que, en gran medida, los resultados dependen mucho de la conservación esquelética y los tipos de enterramiento, es posible afirmar que 
siempre se estará lejos de reconstruir el perfil demográfico real de una población prehistórica.

No obstante, sí ha sido posible corroborar la utilidad de los métodos de estimación paleodemográfica en la medida en que permiten interpretar patrones de enterramiento diferenciales entre tipos de sepulcro, épocas o zonas geográficas, muy posiblemente vinculados a cuestiones culturales o sociales. En el sentido estrictamente metodológico, es evidente que existe una necesidad de unificar criterios de estimación de edad y sexo y, sobre todo, de establecer categorías de edad que permitan trabajar con variables comunes en trabajos de este tipo en la Prehistoria Reciente. Además, un aspecto a tener en cuenta que repite en todos los estudios es la importancia de los individuos subadultos, especialmente infantiles y neonatos, por el papel fundamental que desempeñan en la estructura poblacional arcaica. La correcta identificación de estos grupos de edad permite detectar determinadas anomalías demográficas en la curva de mortalidad, supervivencia o en los valores de la esperanza de vida al nacer, las cuales pueden ser enormemente útiles para entender qué tipo de acceso tenían a la tumba estos grupos de edad. También especial atención merecen todos los sujetos menores de 5 años, cuya ausencia en cementerios colectivos prehistóricos, especialmente en el periodo Neolítico Final-Calcolítico. Esta ausencia parece ser constante en la mayoría de yacimientos, a excepción de San Juan ante Portam Latinam. En este yacimiento el ligero aumento de los individuos subadultos-aunque no llegue a los valores propios del modelo de población arcaico- ofrece una interpretación demográfica que difiere considerablemente de otros enterramientos colectivos coetáneos.

Más allá de los presumibles problemas de conservación de estos restos, se observa que las excavaciones en las que se realiza un registro antropológico exhaustivo de los osarios, tiende a repetirse la infrarrepresentación esquelética de las clases de edad de los más jóvenes. Este fenómeno afecta al análisis demográfico en dos sentidos: por un lado, deforma considerablemente los valores esperados para un tipo de población arcaica en la que la mortalidad infantil es alta, por encima del 200-300\%o (Ledermann, 1969; Bocquet-Appel y Masset, 1977); por otro lado, provoca una sobreestimación en la presencia de otros grupos de edad como consecuencia de esa importante ausencia de individuos infantiles fallecidos. La mayoría de explicaciones aducidas a este fenómeno en el campo de la Antropología Cultural tienen que ver con una pauta muy común en sociedades del pasado en las que los infantes eran excluidos de la esfera social y pública, por lo que es frecuente que su lugar de enterramiento sea distinto que el del resto de la población. Por esta razón se desarrollaron ciertas herramientas de corrección demográfica, algunas de ellas utilizadas en los trabajos que se analizan en este texto. Dichas herramientas suponen un gran avance para medir y comparar las composiciones poblacionales de las tumbas colectivas y, en definitiva, comprobar si realmente restringió el acceso a 
determinados segmentos de población como consecuencia de un estatus social diferenciado.

\section{CONCLUSIONES}

La revisión bibliográfica de los estudios paleodemográficos en la península ibérica que se realiza en este trabajo ha permitido valorar y analizar aquella metodología que mejor se adapta en yacimientos funerarios colectivos de época prehistórica. A lo largo de dicha revisión se ha observado que los parámetros demográficos resultantes de los estudios citados en yacimientos de tipo monumental, tales como los dólmenes, generalmente son acordes con esta hipótesis de reclutamiento selectivo, en los que quizás solo algunos grupos de la población estuvieran destinados a ser enterrados en este tipo de sepulcros. En éstos parece encontrarse una pauta de exclusión más definida que en otro tipo de enterramientos o en épocas más avanzadas, tal y como ocurre en la Edad del Bronce en el Mediterráneo y en Andalucía Oriental.

No obstante, se deberían tener muy en cuenta las dificultades intrínsecas que subyacen al problema paleodemográfico y a las colecciones esqueléticas al tratarse de muestras sesgadas por el paso del tiempo y por las circunstancias propias del contexto funerario. Es por esta razón por la que el análisis de los osarios colectivos debe prestar especial atención a la historia tafonómica del contexto y a la cronología de los enterramientos, con el fin de interpretar el carácter del depósito funerario y no extraer conclusiones demasiado precipitadas sobre el perfil demográfico de las poblaciones arqueológicas.

\section{BibliogRAFÍA}

Acsádi, György y Nemeskéri, János (1970): History of Human Life, Span and Mortality. Budapest: Akadémiai Kiadó.

Alesan, Alícia, Malgosa, Assumpció y Simó, Carles (1999): "Looking into the demography of an Iron Age population in the Western Mediterranean. I. Mortality". American Journal of Physical Anthropology: The Official Publication of the American Association of Physical Anthropologists, 110(3), pp. 285301. $\quad$ https://doi.org/10.1002/(SICI)1096-8644(199911)110:3\%3C285::AIDAJPA3\%3E3.0.CO;2-2 
Angel, J. Lawrence (1969): “The bases of paleodemography”. American Journal of Physical Anthropology, 30(3), pp. 427438. https://doi.org/10.1002/ajpa.1330300314

Aranda, Gonzalo, Lozano, Águeda, Camalich, Ma Dolores, Martín, Dimas, Rodríguez, Francisco J., Trujillo, Aioze, Santana, Jonathan y Clop, Xavier (2017): "La cronología radiocarbónica de las primeras manifestaciones megalíticas en el sureste de la Península Ibérica: las necrópolis de Las Churuletas, La Atalaya y Llano del Jautón (Purchena, Almería)”. Trabajos de Prehistoria, 74 (2), pp. 257277. https://doi.org/10.3989/tp.2017.12194

Armendáriz Gutiérrez, Ángel (2007): “Cronología”. En J. I. Vegas Aramburu (ed.), San Juan ante Portam Latinam: una inhumación colectiva prehistórica en el Valle Medio del Ebro. Memoria de las excavaciones arqueológicas 1985, 1990 y 1991. Vitoria-Gasteiz: Fundación José Miguel de Barandiarán y Diputación Foral de Navarra, pp. 101-103.

Beck, Jess (2016): "Part of the Family: Age, Identity, and Burial in Copper Age Iberia”. En A. J. Osterholtz (ed.), Theoretical Approaches to Analysis and Interpretation of Commingled Human Remains, Bioarchaeology and Social Theory. Cham: Springer, pp. 47-73.

Bello, Silvia y Andrews, Peter (2006): "The intrinsic pattern of preservation of human skeletons and its influence on the interpretation of funerary behaviours". En Gowland, R. y Knüsel, C. (eds.), Social archaeology of funerary remains. Oxford: Oxbow Books, pp. 1-13.

Bocquet-Appel, Jean-Pierre (2002): "Paleoanthropological Traces of a Neolithic Demographic Transition”. Current Anthropology, 43, 4, pp. 637650. https://doi.org/10.1086/342429

Bocquet-Appel, Jean-Pierre y De Miguel Ibáñez, M. Paz. (2002): "Demografía de la difusión neolítica en Europa y los datos paleoantropólogicos”. Sagutum, 5, pp. 2344.

Bocquet-Appel, Jean-Pierre y Masset, Claude (1977): "Estimateurs en paléodémographie”. L’Homme, 4, pp. 65-90. http://www.jstor.org/stable/25159312

Bocquet-Appel, Jean-Pierre y Masset, Claude (1982): "Farewell to Paleodemography". Journal of Human Evolution, 11, pp. 321-333. https://doi.org/10.1016/S00472484(82)80023-7

Brothwell, Don (1972): "Palaeodemography and earlier British populations”. World Archaeology, 4 (1), pp. 5-18. https://doi.org/10.1080/00438243.1972.9979521 
Campillo, Domingo (1995): "Mortalidad y esperanza de vida en la Península Ibérica, desde la Prehistoria a la Edad Media”. En R. Fábregas Valcarce, F. Pérez Losada y C. Fernández Ibáñez (Eds): Arqueoloxía da Morte. Arqueoloxía da Mortena Península Ibérica desde as Orixes ata o Medievo, Xinzo de Limia, pp. 319-340.

Chamberlain, Andrew (2009): “Archaeological Demography”. Human Biology, 81 (3), pp. 275-286. https://doi.org/10.3378/027.081.0309

Delibes de Castro, Germán (1995): "Ritos funerarios, demografía y estructura social entre las comunidades neolíticas de la Submeseta Norte”. En R. Fábregas, F. Pérez, y C. Fernández (Eds.), Arqueología da Morte. Arqueología da Morte na Península Ibérica desde as Orixes ata o Medievo. Xinzo de Limia, pp. 61-94.

Delibes de Castro, Germán (2010): "La investigación de las sepulturas colectivas monumentales del IV milenio A.C. en la Submeseta Norte española. Horizonte 2007”. Munibe (Antropologia-Arkeologia), 32, pp. 12-56.

Domínguez del Triunfo, Helena (2013): Aproximación paleodemográfica a las poblaciones del entorno mediterráneo en la Edad del Bronce (Trabajo de Fin de Máster). Universidad Autónoma de Madrid, Madrid. Disponible en http://hdl.handle.net/10486/660350

Etxeberria, Francisco y Herrasti, Lourdes (2007): "Los restos humanos del enterramiento de San Juan ante Portam Lantinam (Laguardia, Álava): caracterización de la muestra, tafonomía y paleopatología”. En J. I. Vegas (ed.), San Juan ante Portam Latinam: una inhumación colectiva prehistórica en el Valle Medio del Ebro. Memoria de las excavaciones arqueológicas 1985, 1990 y 1991. Vitoria-Gasteiz: Fundación José Miguel de Barandiarán y Diputación Foral de Navarra, pp. 159-280.

Fernández-Crespo, Teresa y De La Rúa, Concepción (2016): “Demographic differences between funerary caves and megalithic graves of northern Spanish Late Neolithic/Early Chalcolithic". American Journal of Physical Anthropology, 160(2), pp. 284-297. https://doi.org/10.1002/ajpa.22963

Fernández-Crespo, Teresa, Schulting, Rick, J., Ordoño, Javier, Duering, Andreas, Etxeberria, Francisco, Herrasti, Lourdes, Armendariz, Ángel y Vegas, José, I. (2018): "New radiocarbon dating and demographic insights into San Juan ante Portam Latinam, a possible Late Neolithic war grave in North-Central Iberia”. American Journal of Physical Antrhopology, 166, pp. 760771. https://doi.org/10.1002/ajpa.23465

Fernández-Eraso, Javier y Mujika-Alustiza, José Antonio (2013): “The megalithic station of the Rioja Alavesa: chronology, origins and utilisation cycles”. Zephyrus, 
71 ,

pp.

89-106.

Disponible

en https://dialnet.unirioja.es/servlet/articulo?codigo $=4457738$

Guerra Doce, Elisa, Delibes de Castro, Germán, Zapatero Magdaleno, Pilar y Villalobos García, Rodrigo (2009): "Primus inter pares: estrategias de diferenciación social en los sepulcros megalíticos de la Submeseta Norte Española”. BSAA arqueología, 75 , pp. 41-65.

Disponible

en https://dialnet.unirioja.es/servlet/articulo?codigo=3627354

Hassan, Fekri A. (1978): “Demographic Archaeology”. En M. Schiffer (ed.), Advances in archaeological method and theory, 1. London: Academic Press, pp. 105-141. Disponible en https://www.jstor.org/stable/20170131

Howell, Nancy (1976): “Toward an Uniformitarian Theory Human Paleodemography”. Journal of Human Evolution, 5, pp. 25-40. https://doi.org/10.1016/00472484(76)90097-X

Ledermann, Sully (1969): Nouvelles tables-types de mortalité. Paris: Presses Universitaires de France. Travaux et Documents, 53.

Jiménez Brobeil, Sylvia A., Robledo, Beatriz y Coronado, Francisco (1991): "Aproximación a la paleodemografía de poblaciones prehistóricas de Andalucía Oriental”. En Actas del II Congreso de Historia de Andalucía. Córdoba: Publicaciones de la Consejería de Cultura y Medio Ambiente de la Junta de Andalucía y obra social y cultural Cajasur, pp. 177-185.

Masset, Claude (1973): "La Démographie des populations inhumées: Essai de paléodemographie". L'Homme, 4(4), 95-131.

Masset, Claude (1986): “Le recrutement d'un ensemble funéraire”. En Duday, H. y Masset, C. (eds.), Anthropologie physiqueetarchéologie: methods d'études des sépultures. Paris: Editions du CNRS, pp. 111134. https://doi.org/10.3406/hom.1973.367382

Olalde, Iñigo, Brace, Selina, Allentoft, Morten E., Armit, Ian, Kristiansen, Kristian, Booth, Thomas, Rohland, Nadin, Mallick, Swapan, Szécsényi-Nagy, Anna, Mittnik, Alissa, Altena, Eveline, Lipson, Mark, Lazaridis, Iosif, Harper, Thomas K., Patterson, Nick, Broomandkhoshbacht, Nasreen, Diekmann, Yoan, Faltyskova, Zuzana, Fernandes, Daniel, Ferry, Matthew, Harney, Eadaoin, de Knijff, Peter, Michel, Megan, Oppenheimer, Jonas, Stewardson, Kristin, Barclay, Alistair, Alt, Kurt Werner, Liesau, Corina, Ríos, Patricia, Blasco, Concepción, Vega Miguel, Jorge, Menduiña García, Roberto, Avilés Fernández, Azucena, Bánffy, Eszter, Bernabò-Brea, Maria, Billoin, David, Bonsall, Clive, Bonsall, Laura, Allen, Tim, Büster, Lindsey, Carver, Sophie, Castells Navarro, Laura, Craig, Oliver E., Cook, Gordon T., Cunliffe, Barry, Denaire, Anthony, Dinwiddy, Kirsten Egging, 
Dodwell, Natasha, Ernée, Michal, Evans, Christopher, Kuchařík, Milan, Farré, Joan Francès, Fowler, Chris, Gazenbeek, Michiel, Garrido Pena, Rafael, HaberUriarte, María, Haduch, Elżbieta, Hey, Gill, Jowett, Nick, Knowles, Timothy, Massy, Ken, Pfrengle, Saskia, Lefranc, Philippe, Lemercier, Olivier, Lefebvre, Arnaud, Heras Martínez, César, Galera Olmo, Virginia, Bastida Ramírez, Ana, Lomba Maurandi, Joaquín, Majó, Tona, McKinley, Jacqueline I., McSweeney, Kathleen, Balázs Gusztáv, Mende, Modi, Alessandra, Kulcsár, Gabriella, Kiss, Viktória, Czene, András, Patay, Róbert, Endrődi, Anna, Köhler, Kitti, Hajdu, Tamás, Szeniczey, Tamás, Dani, János, Bernert, Zsolt, Hoole, Maya, Cheronet, Olivia, Keating, Denise, Velemínský, Petr, Dobeš, Miroslav, Candilio, Francesca, Brown, Fraser, Flores Fernández, Raúl, Herrero-Corral, Ana-Mercedes, Tusa, Sebastiano, Carnieri, Emiliano, Lentini, Luigi, Valenti, Antonella, Zanini, Alessandro, Waddington, Clive, Delibes, Germán, Guerra-Doce, Elisa, Neil, Benjamin, Brittain, Marcus, Luke, Mike, Mortimer, Richard, Desideri,, Jocelyne, Besse, Marie, Brücken, Günter, Furmanek, Mirosław, Hałuszko, Agata, Mackiewicz, Maksym, Rapiński, Artur, Leach, Stephany, Soriano, Ignacio, Lillios, Katina T., Cardoso, João Luís, Parker Pearson, Michael, Włodarczak, Piotr, Price, T. Douglas, Prieto, Pilar, Rey, Pierre-Jérôme, Risch, Roberto, Rojo Guerra, Manuel A., Schmitt, Aurore, Serralongue, Joël, Silva, Ana Maria, Smrčka, Václav, Vergnaud, Luc, Zilhão, João, Caramelli, David, Higham, Thomas, Thomas, Mark G., Kennett, Douglas J., Fokkens, Harry, Heyd, Volker, Sheridan, Alison, Sjögren, Karl-Göran, Stockhammer, Philipp W., Krause, Johannes, Pinhasi, Ron, Haak, Wolfgang, Barnes, Ian, Lalueza-Fox, Carles y Reich, David (2018): “The Beaker phenomenon and the genomic transformation of northwest Europe”. Nature, 555, pp. 190-195. Disponible en https://doi.org/10.1038/nature25738

Ortega Muñoz, Allan (2004): “La paleodemografía: ¿un instrumento para simular el comportamiento demográfico del pasado? Análisis comparativo con la demografía histórica en la Ciudad de México del -siglo XIX”. Estudios Demográficos y Urbanos, 19, 1(55) pp. 181-214. Disponible en http://www.jstor.org/stable/40315175

Roca, María G. (2012): “Paleodemografía: problemas metodológicos y perspectivas”. Cpag, 22, pp. 67-77. en http://revistaseug.ugr.es/index.php/cpag/article/view/2416/2533

Rojo-Guerra, Manuel A., Kunst, Michael, Garrido-Pena, Rafael, García-Martínez de Lagrán, Íñigo y Morán, Guillermo (2005): Un desafío a la eternidad: tumbas monumentales en el valle de Ambrona. Valladolid: Junta de Castilla y León. Arqueología en Castilla y León, Memorias 14

Séguy, Isabelle y Buchet, Luc (2013): Handbook of Palaeodemography. London: Springer International Publishing. 
Sellier, Pascal (1996): “La mise en évidence d'anomalies démographiques et leurinterprétatión: population, recrutement et práctiques funéraires de tumulus de Courtesoult”. En Pinigre, J. F. (ed.), Nécrópoles et societé aun premirâge du Fer: le tumulus de Courtesoult (Haute-Saône). Paris: Maison des Sciences d l'Homme, 54, pp. 188-202.

Silva, Ana Maria (2003): "Portuguese populations of late Neolithic and Chalcolithic periods exhumed from collective burials: an overview”. Anthropologie, 41(1-2), pp. 55-64. Disponible en http://www.jstor.org/stable/26292629

Szecsenyi-Nagy, Anna, Roth, Christina, Brandt, Guido, Rihuete-Herrada, Cristina, Tejedor-Rodríguez, Cristina, Held, Petra, García-Martínez-de-Lagrán, Íñigo, Arcusa Magallón, Héctor, Zesch, Stephanie, Knipper, Corina, Bánffy, Eszter, Friederich, Susanne, Meller, Harald, Bueno Ramírez, Primitiva, Barroso Bermejo, Rosa, de Balbín Behrmann, Rodrigo, Herrero-Corral, Ana M., Flores Fernández, Raúl, Alonso Fernández, Carmen, Jiménez Echevarría, Javier, Rindlisbacher, Laura, Oliart, Camila, Fregeiro, María-Inés, Soriano, Ignacio, Vicente, Oriol, Micó, Rafael, Lull, Vicente, Soler Díaz, Jorge, López Padilla, Juan Antonio, Roca de Togores Muñoz, Consuelo, Hernández Pérez; Mauro S., Jover Maeste, Francisco Javier, Lomba Maurandil, Joaquín, Avilés Fernández, Azucena, Lillios, Katina T., Silva, Ana Maria, Magalhães Ramalho, Miguel, Oosterbeek, Luiz Miguel, Cunha, Claudia, Waterman, Anna J., Roig Buxó, Jordi, Martínez, Andrés, Ponce Martínez, Juana, Hunt Ortiz, Mark, Mejías-García, Juan Carlos, Pecero Espín, Juan Carlos, Cruz-Auñón Briones, Rosario, Tomé, Tiago, Carmona Ballestero, Eduardo, Cardoso, João Luís, Araújo, Ana Cristina, Liesau von Lettow-Vorbeck, Corina, Blasco Bosqued, Concepción, Ríos Mendoza, Patricia, Pujante, Ana, Royo-Guillén, José I., Esquembre Beviá, Marco Aurelio, Dos Santos Goncalves, Victor Manuel, Perreira, Rui, Morán Hernández, Elena, Méndez Izquierdo, Elena, Vega y Miguel, Jorge, Menduiña García, Roberto, Martínez Calvo, Victoria, López Jímenez, Óscar, Krause, Johannes, Pichler, Sandra L., Garrido-Pena, Rafael, Kunst, Michael, Risch, Roberto, Rojo-Guerra, Manuel A., Haak, Wolfgang y Alt, Kurt W. (2017): "The maternal genetic make-up of the Iberian Peninsula between the Neolithic and the Early Bronze Age”. Scientific reports, 7(1), 15644. https://doi.org/10.1038/s41598-017-15480-9

Ubelaker, Douglas (1974): "Reconstruction of Demographic Profiles from Ossuary Skeletal Samples: A Case Study from the Tidewater Potomac". Contributions to Anthropology, 18, pp. 1-79. https://doi.org/10.5479/si.00810223.18.1

Waterman, Anna. J. y Thomas, Jonathan. T. (2011): "When the bough breaks: Childhood mortality and burial practice in late neolithic Atlantic Europe”. Oxford Journal of Archaeology, 30(2), pp. 165-183. https://doi.org/10.1111/j.14680092.2011.00363.x 
Weiss, Kenneth M. y Martin Wobst, H. (1973): "Demographic models for Anthropology". Memoirs of the Society for American Archaeology, 27, pp. 1186. https://www.jstor.org/stable/pdf/25146719.pdf

Welinder, Stieg (1979): "Prehistoric Demography". Lund: Acta Archaeologica Ludensia. Series in $8^{\circ}$ Minore. No 8.

Wood, James W., Milner, George R., Harpending, Henry C. y Weiss, Kenneth M. (1992): "The Osteological Paradox Problems of Inferring Prehistoric Health from Skeletal Samples”. Current Anthropology, 33(4), pp. 343-370. 